\title{
Valores intermediários de BNP são capazes de predizer eventos em pacientes com síndrome coronária aguda?
}

\author{
Intermediate levels of BNP were related with cardiology events \\ in acute coronary syndromes?
}

\author{
Alexandre de Matos Soeiro ${ }^{1}$, Eduardo A. de Castro Roque ${ }^{2}$, Priscila Galvão Póvoa ${ }^{1}$, Aline Siqueira \\ Bossa $^{1}$, Cindel Nogueira Zullino ${ }^{1}$, Maria Carolina Feres de Almeida Soeiro ${ }^{1}$, Tatiana de \\ Carvalho Andreucci Torres Leal ${ }^{1}$, Yuri Justi Jardim ${ }^{1}$, Mariana Marques Sinigaglia ${ }^{1}$, Alex \\ França da Silva ${ }^{1}$, Bruna Muntaneli ${ }^{1}$, Carlos V. Serrano Jr. ${ }^{1}$, Múcio Tavares de Oliveira Jr. ${ }^{1}$
}

Soeiro AM, Roque EAC, Póvoa PG, Bossa AS, Zullino CN, Soeiro MCFA, Leal TCAT, Jardim YJ, Sinigaglia MM, Silva AF, Muntaneli B, Serrano Jr. CV, Oliveira Jr MT. Valores intermediários de BNP são capazes de predizer eventos em pacientes com síndrome coronária aguda? Intermediate levels of BNP were related with cardiology events in acute coronary syndromes? Rev Med (São Paulo). 2016 abr.-jun.;95(2):53-9.

RESUMO: Introdução: Diversos estudos na literatura têm relacionado valores elevados de peptídeo natriurético cerebral (BNP) com pior prognóstico em pacientes com síndrome coronária aguda (SCA). No entanto, valores entre $100 \mathrm{pg} / \mathrm{mL}$ e $400 \mathrm{pg} / \mathrm{mL}$ são considerados limítrofes e ainda questionados em relação à diagnóstico e ocorrência de eventos. Métodos: Trata-se de estudo retrospectivo observacional com objetivo de avaliar se o valor intermediário de BNP na admissão hospitalar é capaz de predizer prognóstico intrahospitalar. Os pacientes foram divididos em dois grupos: grupo I: $\mathrm{BNP} \leq 100 \mathrm{pg} / \mathrm{mL}$; grupo II: $100<\mathrm{BNP}$ $<400 \mathrm{pg} / \mathrm{mL}$. Foram incluídos 405 pacientes (235 no grupo I e 170 no grupo II) com SCA. Obtiveram-se dados referentes à comorbidades e medicações utilizadas. Análise estatística: $\mathrm{O}$ desfecho primário foi mortalidade por todas as causas. O desfecho secundário foi eventos combinados (choque cardiogênico, reinfarto, morte, acidente vascular cerebral e sangramento). A comparação entre grupos foi realizada através de Q-quadrado e ANOVA. A análise multivariada foi realizada por regressão logística, sendo considerado significativo $p<0,05$. Resultados: $\mathrm{Na}$ comparação entre os grupos I e II, observaram-se diferenças em relação à prevalência de diabetes mellitus e angioplastia coronária prévia. Na análise multivariada, observaram-se diferenças significativas entre os grupos I e II em relação à ocorrência de choque cardiogênico $(2,55 \%$ x $10,59 \%, \mathrm{OR}=4,09, p=0,01)$, respectivamente. Conclusão: Valores intermediários de BNP não foram capazes de predizer mortalidade em pacientes com SCA. No entanto, observou-se uma maior incidência de choque cardiogênico.

Descritores: Peptídeo natriurético cerebral; Prognóstico; Síndrome coronariana aguda.

\footnotetext{
1. Instituto do Coração (InCor) do Hospital das Clínicas da Universidade de São Paulo, Unidade de Emergência. Emails: prigpovoa@ hotmail.com, aline.bossa@incor.usp.br, cnzullino@gmail.com, carolferes@hotmail.com, tati torres@hotmail.com,justijardim@gmail. com,mmsinigaglia@gmail.com, alex_fran@hotmail.com, bru.muntanelli@gmail.com,cvserranojr@gmail.com, mucio@incor.usp.br 2. Hospital Metropolitano, Unidade Coronariana - Serra, ES - Brasil. Email: eduardo.castro.roque@gmail.com

Autor para correspondência: Alexandre de Matos Soeiro. Av. Dr. Eneas de Carvalho Aguiar, 44. CEP: 05403-900 - Cerqueira César, São Paulo, Brasil. E-mail: alexandre.soeiro@bol.com.br.
} 
ABSTRACT: Introduction: Several studies in the literature have linked high levels of brain natriuretic peptide (BNP) with poor prognosis in patients with acute coronary syndrome (ACS). However, values between $100 \mathrm{pg} / \mathrm{ml}$ and $400 \mathrm{pg} / \mathrm{ml}$ are considered borderline and also questioned about the occurrence of events and diagnosis. Methods: This is an observational retrospective study to evaluate the BNP intermediate value at hospital admission can predict in-hospital prognosis. The patients were divided into two groups: Group I: BNP $<100$ pg/ml; Group II: $100<$ BNP $<$ $400 \mathrm{pg} / \mathrm{mL}$. It included 405 patients (235 in group I and 170 in group II) with ACS. Data were obtained regarding comorbidities and medications used. Statistical analysis: The primary outcome was mortality from all causes. The secondary endpoint was combined events (cardiogenic shock, reinfarction, death, stroke

\section{INTRODUÇÃO}

$\mathrm{O}$ coração secreta dois peptídeos natriuréticos principais: o peptídeo natriurético atrial (ANP), que é sintetizado nos átrios, e o peptídeo natriurético cerebral (BNP), que é produzido pelos ventrículos. Ambos são liberados na circulação sanguínea em reposta à distensão do músculo cardíaco, levando à vasodilatação, natriurese e inibição da ativação simpática e do sistema renina-angiotensina-aldosterona ${ }^{1}$. O BNP tem sido utilizado como marcador de escolha associado à risco de morte $\mathrm{e}$ eventos em pacientes com doenças cardiovasculares como insuficiência cardíaca ou síndromes coronárias agudas (SCA). Embora a fisiopatologia desse achado não esteja completamente esclarecida, concentrações elevadas de BNP em SCA mostraram ser preditoras de eventos em curto e longo prazo $^{1-6}$.

Diversos estudos apresentaram valores de corte únicos através dos quais o BNP encontrou-se relacionado a piores desfechos em $\mathrm{SCA}^{6-16}$. No entanto, a análise específica de valores intermediários ainda não foi descrita. Nesse contexto, realizamos este estudo com o objetivo de avaliar se o valor intermediário de BNP na admissão hospitalar é capaz de predizer prognóstico intrahospitalar em SCA.

\section{MÉTODOS}

\section{População do estudo}

Trata-se de estudo retrospectivo e observacional. Incluíram-se 405 pacientes com SCA (com [19,5\%] e sem supradesnível de ST [80,5\%]) admitidos entre maio de 2010 a maio de 2014 no setor de emergência. Os pacientes foram divididos em dois grupos: grupo I: $\mathrm{BNP} \leq 100 \mathrm{pg} / \mathrm{mL}(\mathrm{N}=$ 235); grupo II: $100<\mathrm{BNP}<400 \mathrm{pg} / \mathrm{mL}(\mathrm{N}=170)$. Foram excluídos pacientes com BNP $>400 \mathrm{pg} / \mathrm{mL}$ e aqueles em classificação Killip III/IV na admissão hospitalar.

Consideraram-se como SCA todos os pacientes que preencheram os critérios estabelecidos pela última and bleeding). The comparison between groups was performed using Q-square test and ANOVA. Multivariate analysis was performed by logistic regression, considering significant $p<0.05$. Results: Comparing the groups I and II, there were differences in the prevalence of diabetes and previous coronary angioplasty. In multivariate analysis, there were significant differences between groups I and II in the occurrence of cardiogenic shock $(2.55 \%$ vs. $10.59 \%$, OR $=4.09, p=0.01$ ), respectively. Conclusion: Intermediate BNP values were not able to predict mortality in patients with ACS. However, there was a higher incidence of cardiogenic shock.

Keywords: Natriuretic peptide, Brain; Prognosis, Acute coronary syndrome.

diretriz da American Heart Association ${ }^{17-19}$. SCA com supradesnível do segmento ST foi definida como presença de dor torácica com alteração persistente do segmento $\mathrm{ST} \geq 0,1 \mathrm{mV}$ nas derivações do plano frontal e $\geq 0,2 \mathrm{mV}$ nas derivações precordiais, em pelo menos 2 derivações contíguas. SCA sem supradesnível de ST foi definida como presença de dor torácica associadas à alterações eletrocardiográficas ou elevação/queda de troponina na internação ou, na ausência destes, quadro clínico e fatores de risco compatíveis com angina instável (dor torácica ao repouso ou aos mínimos esforços, severa ou ocorrendo em padrão em crescendo). Sangramento maior foi definido pelo escore de $B A R C^{20}$ tipos 3 e 5 e sangramento menor pelos tipos 1 e 2 . Reinfarto foi considerado quando houve recorrência da dor torácica associada à nova elevação de troponina. Acidente vascular encefálico isquêmico foi considerado quando o paciente apresentasse novo déficit neurológico focal motor confirmado através de tomografia computadorizada de crânio.

Os seguintes dados foram obtidos: idade, sexo, presença de diabetes mellitus, hipertensão arterial sistêmica, tabagismo, dislipidemia, história familiar para doença coronária precoce, doença arterial coronária prévia (angioplastia ou cirurgia de revascularização miocárdica anterior), acidente vascular cerebral prévio, hematócrito, creatinina, pico de troponina, fração de ejeção do ventrículo esquerdo, pressão arterial sistólica e medicações utilizadas nas primeiras 24 horas da internação.

A dosagem de BNP foi adquirida imediatamente após a admissão hospitalar do paciente e analisada pelo kit Abbott Axsym 5.0 system (Abbott Laboratories, Abbott Park, IL). Para quantificação da troponina I, foi utilizado o kit comercial ADVIA Centaur ${ }^{\circledR}$ TnI-Ultra (Siemens Healthcare Diagnostics, Tarrytown, NY, USA) em equipamento automatizado da mesma marca. O valor de percentil $99 \%$ é de $0,04 \mathrm{ng} / \mathrm{ml}$.

O trabalho foi submetido e aprovado pelo comitê de ética e pesquisa. O termo de consentimento livre e 
esclarecido foi preenchido por todos os pacientes incluídos no estudo.

\section{Análise estatística}

O desfecho primário foi mortalidade intrahospitalar por todas as causas. $\mathrm{O}$ desfecho secundário foi eventos combinados (choque cardiogênico, reinfarto, morte, AVCI e sangramento) no período intrahospitalar. A comparação entre grupos foi realizada através de Q-quadrado para as variáveis categóricas e ANOVA para as variáveis contínuas. A análise multivariada foi realizada por regressão logística. Foi considerado significativo $p<0,05$. Foram incluídas como variáveis na análise todas as características basais apresentadas na tabela $1 \mathrm{com}$ diferença significativa entre os grupos.

\section{RESULTADOS}

As características basais da população estudada de acordo com o valor de BNP encontram-se na Tabela 1. Na comparação entre os Grupos I e II, observaram-se diferenças em relação à prevalência de diabetes mellitus $(34,80 \% \times 48,82 \%, p=0,005)$ e angioplastia coronária prévia $(27,66 \% \times 18,24 \%, p=0,028)$, respectivamente.

Os resultados da análise multivariada comparando diferentes desfechos intrahospitalares entre os grupos de acordo com o valor de BNP à admissão são apresentados na Tabela 2 e Figura 1. Observaram-se diferenças significativas entre os Grupos I e II somente em relação à ocorrência de choque cardiogênico $(2,55 \%$ x 10,59\%, OR $=4,09, p=0,01)$, respectivamente.

Tabela 1. Características clínicas basais de pacientes com diferentes níveis de PAS na admissão hospitalar

\begin{tabular}{|c|c|c|c|}
\hline & $\mathrm{BNP} \leq 100 \mathrm{pg} / \mathrm{ml}$ & $100<\mathrm{BNP}<400 \mathrm{pg} / \mathrm{ml}$ & $p$ \\
\hline Idade (média) & 61,2 & 65,6 & 0,22 \\
\hline Sexo masculino (\%) & $60 \%$ & $59 \%$ & 0,97 \\
\hline Diabetes Mellitus (\%) & $35 \%$ & $49 \%$ & 0,005 \\
\hline HAS (\%) & $76 \%$ & $84 \%$ & 0,051 \\
\hline Tabagismo (\%) & $43 \%$ & $37 \%$ & 0,21 \\
\hline HF Positiva para DAC (\%) & $15 \%$ & $11 \%$ & 0,16 \\
\hline Dislipidemia (\%) & $50 \%$ & $47 \%$ & 0,53 \\
\hline IC $(\%)$ & $7 \%$ & $11 \%$ & 0,12 \\
\hline AVC prévio (\%) & $6 \%$ & $11 \%$ & 0,08 \\
\hline IAM prévio (\%) & $40 \%$ & $41 \%$ & 0,9 \\
\hline CRM prévia (\%) & $20 \%$ & $19 \%$ & 0,8 \\
\hline ATC prévia (\%) & $28 \%$ & $18 \%$ & 0,028 \\
\hline Ht (\%) (mediana) & 42,9 & 41,1 & 0,24 \\
\hline Pico de troponina (média) $(\mathrm{ng} / \mathrm{ml})$ & 13.2 & 11.8 & 0,32 \\
\hline Cr (mg/dL) (média) & 2,29 & 2,73 & 0,83 \\
\hline PAS (mmHg) (média) & 135,6 & 133,4 & 0,11 \\
\hline FEVE (\%) (média) & 42,2 & 41,2 & 0,1 \\
\hline AAS $(\%)$ & $96 \%$ & $97 \%$ & 0,87 \\
\hline B-bloqueador (\%) & $69 \%$ & $65 \%$ & 0,37 \\
\hline Enoxaparina (\%) & $77 \%$ & $68 \%$ & 0,06 \\
\hline $\operatorname{IECA}(\%)$ & $52 \%$ & $49 \%$ & 0,48 \\
\hline Estatina (\%) & $85 \%$ & $84 \%$ & 0,15 \\
\hline
\end{tabular}

Legenda: $\mathrm{PAS}=$ pressão arterial sistólica; $\mathrm{HAS}=$ hipertensão arterial sistêmica; $\mathrm{HF}=$ história familiar; DAC=doença arterial coronária; IC=insuficiência cardíaca; IAM=infarto agudo do miocárdio; $C R M=$ cirurgia de revascularização miocárdica; TC=angioplastia coronária; $\mathrm{Ht}=$ hematócrito; $\mathrm{Cr}=$ creatinina; $\mathrm{FEVE}=$ fração de ejeção do ventrículo esquerdo; $\mathrm{IECA}=$ inibidor da enzima conversora de angiotensina; $\mathrm{AVC}=$ acidente vascular cerebral. 


$$
p=0,14
$$

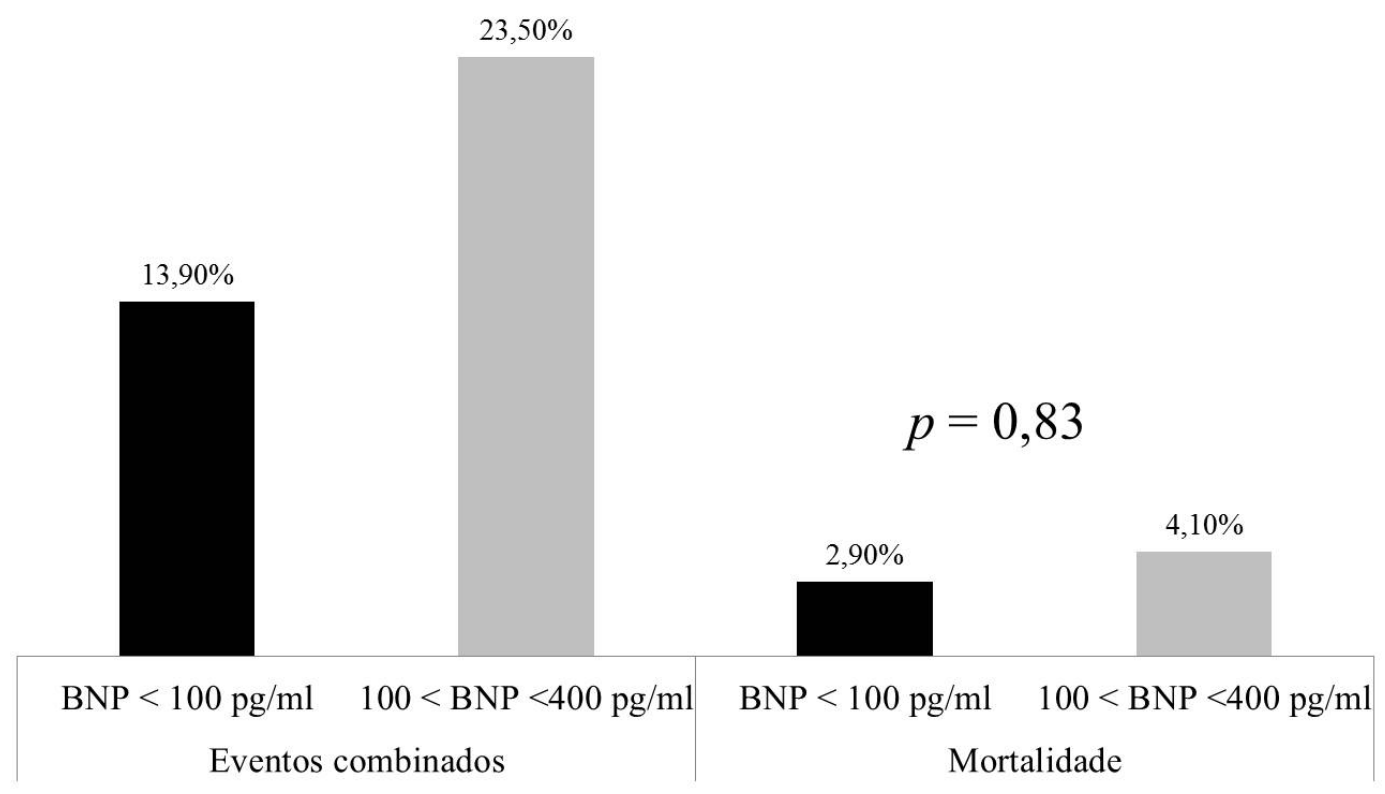

Figura 1. Mortalidade e eventos combinados de acordo com o valor de BNP encontrado

Tabela 2. Resultados da análise multivariada comparando diferentes desfechos intrahospitalares entre os grupos de

acordo com o valor de BNP à admissão

\begin{tabular}{lccccc}
\hline & $\mathbf{B N P} \leq \mathbf{1 0 0} \mathbf{~ p g} / \mathbf{m l}$ & $\mathbf{1 0 0}<\mathbf{B N P}<\mathbf{4 0 0} \mathbf{~ p g} / \mathbf{m l}$ & OR & IC 95\% & $\boldsymbol{p}$ \\
\hline Reinfarto & $0,8 \%$ & $0,5 \%$ & 1,17 & $0,89-15,42$ & 0,9 \\
Choque cardiogênico & $2,6 \%$ & $10,6 \%$ & 4,09 & $1,39-12,03$ & 0,01 \\
Sangramento & $6,8 \%$ & $7,1 \%$ & 1,19 & $0,48-2,98$ & 0,7 \\
AVCI & $0,8 \%$ & $1,2 \%$ & 0,83 & $0,10-6,81$ & 0,87 \\
Mortalidade & $2,9 \%$ & $4,1 \%$ & 1,15 & $0,31-4,24$ & 0,83 \\
Eventos combinados & $13,9 \%$ & $23,5 \%$ & 1,61 & $0,85-3,03$ & 0,14 \\
\hline
\end{tabular}

Legenda: $\mathrm{AVCI}=$ acidente vascular cerebral isquêmico; $\mathrm{OR}=\mathrm{Odds}$ ratio; $\mathrm{IC}=$ intervalo de confiança.

\section{DISCUSSÃO}

Este estudo demonstrou que valores intermediários de BNP, restritos a uma determinada faixa não se correlacionaram com pior prognóstico em pacientes com SCA referente à mortalidade e eventos combinados. Diferentes de outros trabalhos, incluímos pacientes com SCA com supradesnível de ST, algo pouco relatado. Além disso, encontramos tendência importante à ocorrência de maior mortalidade e eventos combinados em valores de BNP intermediários, mas com diferença significativa especificamente em relação à incidência de choque cardiogênico, algo não descrito anteriormente.

Estudo realizado por Azevedo et al. ${ }^{6}$ já apontava que mesmo em valores reduzidos, o BNP poderia ser um marcador importante em pacientes com SCA. Avaliando 125 pacientes com dor torácica e comparando o resultado no BNP com a cintilografia de perfusão miocárdica, os autores demonstraram um índice de acurácia de $66 \%$ relacionado ao BNP para detecção de isquemia quando este era maior que $80 \mathrm{pg} / \mathrm{mL}$. Além disso, acima desse valor, existia clara relação com a mortalidade $(R R=7,29$, 
$p=0,045)^{6}$. De maneira muito semelhante, outro estudo mostrou em 723 pacientes com dor torácica e/ou SCA sem supradesnível de ST que o risco de morte quando o BNP é maior que $101 \mathrm{pg} / \mathrm{mL}$ é 13 vezes maior em 30 dias $(p<$ $0,0001)$ e 5,3 vezes maior em um ano $(p<0,0001)^{21}$.

Ainda avaliando dor torácica, Brown et al. ${ }^{22}$ conseguiram demonstrar maior sensibilidade na detecção de infarto agudo do miocárdio e SCA quando o valor de $\mathrm{BNP}$ era superior à apenas $31 \mathrm{pg} / \mathrm{ml}(p<0,001)$, inclusive com maior relação na ocorrência de eventos em 30 dias $(p=0,004)^{22}$.

Na mesma linha dos estudos anteriores e avaliando valores reduzidos de BNP, trabalho publicado em 2.003 incluindo 1.676 pacientes com SCA sem supradesnível de ST mostrou que a dosagem de BNP acima de $80 \mathrm{pg} / \mathrm{ml}$ esteve fortemente relacionada à morte em 7 dias $(2,5 \% \mathrm{x}$ $0,7 \%, p=0,006)$ e em 6 meses $(8,4 \% \times 1,8 \%, p<0,0001)$. Além disso, a correlação também foi demonstrada em relação à chance do paciente desenvolver insuficiência cardíaca em 30 dias $(5,9 \%$ x 1,0\%, $p<0,001)$, dado esse que corrobora parcialmente nosso achado precoce de maior incidência de choque cardiogênico devido à disfunção ventricular aguda ${ }^{7}$.

De maneira geral, estes trabalhos anteriores apresentam resultados semelhantes aqueles encontrados neste estudo, principalmente pelo fato de usarem cortes de BNP baixos e já apresentarem correlação com prognóstico significativa? .

No entanto, outros estudos usaram ou encontraram valores mais altos de BNP para demonstrar relação com prognóstico em SCA. Scotti et al. ${ }^{8}$ acompanharam 40 pacientes com SCA sem supradesnível de ST e troponina positiva e mensuraram o BNP na chegada e após 96 horas da admissão. Usando um cutoff de $300 \mathrm{pg} / \mathrm{mL}$, os autores mostraram que o BNP foi forte preditor de mortalidade em quatro anos de seguimento com área sob a curva de 0,92. Além disso, demonstrou-se um risco relativo de morte 7,4 vezes maior naqueles com $\mathrm{BNP}$ acima de $300 \mathrm{pg} / \mathrm{mL}(p<0,01)^{8}$.

Outro estudo publicado em 2.009 incluindo 442 pacientes com SCA mostrou no seguimento de dez meses que dosagem de BNP acima de $239 \mathrm{pg} / \mathrm{mL}$ estavam significativamente associadas à maior incidência de morte, readmissão ou insuficiência cardíaca $(\mathrm{OR}=5,86$; IC 95\%: 1,04-32,94 $)^{23}$. Apesar de usar valores mais elevados para avaliação esse é outro estudo que demonstra maior ocorrência de insuficiência cardíaca no seguimento, apresentando certa correlação com nossos achados de choque cardiogênico e talvez com a extensão da perda muscular miocárdica.

Haaf et al. ${ }^{24}$ mostraram em estudo multicêntrico e prospectivo em 1.075 pacientes com dor torácica que BNP maior que $224 \mathrm{pg} / \mathrm{mL}$ esteve relacionado à maior chance de diagnóstico de infarto agudo do miocárdio, com área sob a curva de 0,74. Além disso, o uso de BNP em conjunto com a troponina melhorou a discriminação de pacientes de real alto risco mostrando maior índice de mortalidade em 30 dias $^{24}$.

Estudo mais recente com 600 indivíduos com SCA sem supradesnível de ST seguidos por 13,4 meses mostrou que a dosagem de BNP na admissão do paciente é capaz de predizer morte intrahospitalar (OR $=3,56$; IC 95\%: $1,75-7,23$ ) e em longo prazo ( $\mathrm{HR}=2,46$; IC 95\%: $1,94-$ 3,12 ) de maneira significativa. Além disso, incrementa a classificação de risco usual de TIMI e GRACE ${ }^{25}$.Tal achado é pior prognóstico intrahospitalar é incomum em outros estudos e vem de frente com nossos resultados, apesar de mostrarmos apenas correlação significativa com choque cardiogênico e forte tendência em relação à mortalidade.

Seguindo essa mesma linha de incremento em prognóstico auxiliar aos escores, outro estudo avaliou 449 com SCA com e sem supradesnível de ST em relação à ocorrência de eventos (morte, insuficiência cardíaca e readmissão) em 10 meses utilizando BNP e o escore GRACE. O BNP quando maior que $247 \mathrm{pg} / \mathrm{mL}$ mostrou estar relacionado à maior ocorrência de eventos $(\mathrm{OR}=2,28$; IC 95\%: 1,15-4,51), porém o escore GRACE apresentou apenas resultados limítrofes (OR =2,4; IC 95\%: 0,76-7,56). No entanto, quando usados BNP e escore GRACE juntos, o resultado apresentou um ganho significativo na predição de prognóstico (OR = 6,0; IC 95\%: 2,4-14, 83) 26,27 .

Por último, alguns estudos mostraram adicionalmente relação entre BNP e números de vasos acometidos em pacientes com $\mathrm{SCA}^{28,29}$. Palazzuoli et al. ${ }^{28}$, por exemplo, mostraram que a elevação de BNP era linear em relação ao padrão arterial coronário do paciente, com valores de $86,2 \mathrm{pg} / \mathrm{mL}, 127 \mathrm{pg} / \mathrm{mL}$ e $295 \mathrm{pg} / \mathrm{mL}$ respectivamente associados à padrões uniarteriais, biarteriais ou triarteriais encontrados ${ }^{28}$.

Dessa forma, a associação entre BNP e prognóstico em SCA é clara e tal marcador pode ser utilizado como um incremento na estratificação e risco desses pacientes. Porém, diferentes trabalhos são rotineiramente publicados usando valores diferentes de corte relacionados a prognóstico em diferentes populações.

Este estudo apresenta limitações principalmente pelo fato de ser retrospectivo. No entanto, apresenta resultados que condizem com outros trabalhos e com as recomendações atuais em diretrizes sobre SCA. Apesar de incluirmos pacientes com SCA com supradesnível de ST, algo pouco relatado na literatura, a análise separada desses pacientes não possível devido à casuística. Além disso, o valor médio encontrado de creatinina dos pacientes foi elevado, o que pode superestimar valores de BNP mesmo na ausência de doença cardiovascular significativa.

\section{CONCLUSÃO}

Valores intermediários de BNP não foram capazes de predizer mortalidade em pacientes com SCA. No entanto, observou-se uma maior incidência de choque cardiogênico. 


\section{REFERÊNCIAS}

1. Gaggin HK, Januzzi JL Jr. Natriuretic peptides in heart failure and acute coronary syndrome. Clin Lab Med. 2014;34(1):4358. doi: 10.1016/j.cll.2013.11.007.

2. Futterman LG, Lemberg L. Novel markers in the acute coronary syndrome: BNP, IL-6, PAPP-A. Am J Crit Care. 2002;11(2):168-72. Available from: http://ajcc.aacnjournals. org/content/11/2/168.long.

3. Ndrepepa G, Braun S, Mehilli J, Niemöller K, Schömig A, Kastrati A. A prospective cohort study of prognostic power of N-terminal probrain natriuretic peptide in patients with non-ST segment elevation acute coronary syndromes. Clin Res Cardiol. 2007;96(1):30-7. doi: 10.1007/s00392-0060457-9.

4. Nicholls MG, Frampton CM, Yandle TG. BNP: not just for heart failure. Heart. 2008;94(1):6-7. doi:10.1136/ hrt.2007.119925.

5. León de la Fuente R, Naesgaard PA, Nilsen ST, Woie L, Aarsland T, Gallo P, et al. B-type natriuretic peptide and high sensitive C-reactive protein predict 2 -year all cause mortality in chest pain patients: a prospective observational study from Salta, Argentina. BMC Cardiovasc Disord. 2011;11:57. doi: 10.1186/1471-2261-11-57.

6. Azevedo JC, Reis BC, Barreto NM, F Junior DS, Prezotti LS, Procaci VR, et al. BNP was associated with ischemic myocardial scintigraphy and death in patients at chest pain unit. Arq Bras Cardiol. 2015;104(1):16-23. doi: 10.5935/ abc. 20140175 .

7. Morrow DA, de Lemos JA, Sabatine MS, Murphy SA, Demopoulos LA, DiBattiste PM, et al. Evaluation of B-type natriuretic peptide for risk assessment in unstable angina/ non-ST-elevation myocardial infarction: B-type natriuretic peptide and prognosis in TACTICS-TIMI 18. J Am Coll Cardiol. 2003;41(8):1264-72. http://dx.doi.org/10.1016/ S0735-1097(03)00168-2

8. Scotti AV, Tura BR, Rocha RG, Albuquerque DC. Prognostic value of B-type natriuretic peptide in the mortality of patients with acute coronary syndrome. Arq Bras Cardiol. 2012;99(1):605-12. http://dx.doi.org/10.1590/ S0066-782X2012005000050.

9. Yamamoto T, Sato N, Yasutake M, Takagi H, Morita $\mathrm{N}$, Akutsu K, et al. B-type natriuretic peptide as an integrated risk marker in non-ST elevation acute coronary syndromes. Int J Cardiol. 2006;111(2):224-30. http://dx.doi. org/10.1016/j.ijcard.2005.07.010.

10. Brügger-Andersen T, Pönitz V, Staines H, Pritchard D, Grundt H, Nilsen DW. B-type natriuretic peptide is a long-term predictor of all-cause mortality, whereas highsensitive C-reactive protein predicts recurrent short-term troponin $\mathrm{T}$ positive cardiac events in chest pain patients: a prognostic study. BMC Cardiovasc Disord. 2008;8:34. doi: 10.1186/1471-2261-8-34.

11. Richards AM, Nicholls MG, Espiner EA, Lainchbury JG, Troughton RW, Elliott J, et al. B-type natriuretic peptides and ejection fraction for prognosis after myocardial infarction. Circulation. 2003;107(22):2786-92. http://dx.doi. org/10.1161/01.CIR.0000070953.76250.B9.

12. Sabatine MS, Morrow DA, de Lemos JA, Gibson CM, Murphy SA, Rifai N, et al. Multimarker approach to risk stratification in non-ST elevation acute coronary syndromes: simultaneous assessment of troponin I, C-reactive protein, and B-type natriuretic peptide. Circulation. 2002;105(15):1760-3. http:// dx.doi.org/10.1161/01.CIR.0000015464.18023.0A.

13. Omland T, Persson A, Ng L, O’Brien R, Karlsson T, Herlitz J, Hartford M, et al. N-terminal pro-B-type natriuretic peptide and long-term mortality in acute coronary syndromes. Circulation. 2002;106(23):2913-8. http://dx.doi. org/10.1161/01.CIR.0000041661.63285.AE.

14. Sun T, Wang L, Zhang Y. Prognostic value of B-type natriuretic peptide in patients with acute coronary syndromes. Arch Med Res. 2006;37(4):502-5. http://dx.doi.org/10.1016/j. arcmed.2005.09.007.

15. Ang DS, Kong CF, Kao MP, Struthers AD. Serial bedside B-type natriuretic peptide strongly predicts prognosis in acute coronary syndrome independent of echocardiographic abnormalities. Am Heart J. 2009;158(1):133-40. doi: 10.1016/j.ahj.2009.04.024.

16. Grabowski M, Filipiak KJ, Karpinski G, Wretowski D, Rdzanek A, Huczek Z, et al. Serum B-type natriuretic peptide levels on admission predict not only short-term death but also angiographic success of procedure in patients with acute ST-elevation myocardial infarction treated with primary angioplasty. Am Heart J. 2004;148(4):655-62. http://dx.doi. org/10.1016/j.ahj.2004.04.023.

17. O'Gara PT, Kushner FG, Ascheim DD, et al. 2013 ACCF/AHA Guideline for the Management of ST-Elevation Myocardial Infarction : A Report of the American College of Cardiology Foundation/American Heart Association Task Force on Practice Guidelines. Circulation. 2013;127: e362-e425. doi: 10.1002/ccd.24776.

18. Jneid H, Anderson JL, Wright RS, et al. 2012 ACCF/AHA focused update of the guideline for the management of patients with unstable angina/non-ST-elevation myocardial infarction (updating the 2007 guideline and replacing the 2011 focused update): a report ofthe American College of Cardiology Foundation/American Heart Association Task Force on Practice Guidelines. Circulation. 2012;126:875910. doi: 10.1016/j.jacc.2012.06.004

19. Piegas LS, Timerman A, Feitosa GS, Nicolau JC, Mattos LAP, Andrade MD, et al. V Diretriz da Sociedade Brasileira de Cardiologia sobre Tratamento do Infarto Agudo do Miocárdio com Supradesnível do Segmento ST. Arq Bras Cardiol. 2015;105(2):1-105. doi: 10.5935/abc.20150107.

20. Mehran R, Rao SV, Bahht DL, Gibson M, Caixeta A, Eikelboom J, et al. Standardized Bleeding Definitions for Cardiovascular Clinical Trials. A Consensus Report From the Bleeding Academic Research Consortium. Circulation. 2011;123:2736-2747. http://dx.doi.org/10.1161/ CIRCULATIONAHA.110.009449. 
21. Bassan R, Tura BR, Maisel AS. B-type natriuretic peptide: a strong predictor of early and late mortality in patients with acute chest pain without ST-segment elevation in the emergency department. Coron Artery Dis. 2009;20(2):143-9. doi: 10.1097/MCA.0b013e3283292ac6.

22. Brown AM, Sease KL, Robey JL, Shofer FS, Hollander JE. The impact of B-type natriuretic peptide in addition to troponin I, creatine kinase-MB, and myoglobin on the risk stratification of emergency department chest pain patients with potential acute coronary syndrome. Ann Emerg Med. 2007;49(2):153-63. http://dx.doi.org/10.1016/j. annemergmed.2006.08.024.

23. Ang DS, Welsh P, Watt P, Nelson SM, Struthers A, Sattar N. Serial changes in adiponectin and BNP in ACS patients: paradoxical associations with each other and with prognosis. Clin Sci (Lond). 2009;117(1):41-8. doi: 10.1042/ CS20080506.

24. Haaf P, Reichlin T, Corson N, Twerenbold R, Reiter M, Steuer $\mathrm{S}$, et al. B-type natriuretic peptide in the early diagnosis and risk stratification of acute chest pain. Am J Med. 2011;124(5):444-52. doi: 10.1016/j.amjmed.2010.11.012.

25. García-Alvarez A, Regueiro A, Hernández J, Kasa G, Sitges $\mathrm{M}$, Bosch X, et al. Additional value of B-type natriuretic peptide on discrimination of patients at risk for mortality after a non-ST-segment elevation acute coronary syndrome.
Eur Heart J Acute Cardiovasc Care. 2014;3(2):132-40. doi: $10.1177 / 2048872614520753$.

26. Ang DSC, Wei L, Kao MPC, Lang CC, Struthers AD. A comparison between B-type natriuretic peptide, Global Registry of Acute Coronary Events (GRACE) score and their combination in ACS risk stratification. Heart 2009;95:183642. doi: 10.1136/hrt.2008.160234.

27. Guidez T, Mare'chaux S, Pincon C, Lamour H, Barrailler $\mathrm{S}$, Decourcelle V, et al. Addition of B-type natriuretic peptide to the GRACE score to predict outcome in acute coronary syndrome: a retrospective (development) and prospective (validation) cohort-based study. Emerg Med J. 2012;29:274e279. doi: 10.1136/emj.2010.104422.

28. Palazzuoli A, Maisel A, Caputo M, Fineschi M, Quatrini I, Calabrò A, et al. B-type natriuretic peptide levels predict extent and severity of coronary disease in non-ST elevation coronary syndromes and normal left ventricular systolic function. Regul Pept. 2011;167(1):129-33. doi: 10.1016/j. regpep.2010.12.011.

29. Palazzuoli A, Gennari L, Calabria P, Quatrini I, Vecchiato L, De Paola V, et al. Relation of plasma brain natriuretic peptide levels in non-ST-elevation coronary disease and preserved systolic function to number of narrowed coronary arteries. Am J Cardiol. 2005;96(12):1705-10. http://dx.doi. org/10.1016/j.amjcard.2005.07.094. 